\title{
Resection following concurrent chemotherapy and high-dose radiation for stage IIIA non-small cell lung cancer
}

Jessica S. Donington, MD, ${ }^{\mathrm{a}}$ Rebecca Paulus, BS, ${ }^{\mathrm{a}}$ Martin J. Edelman, MD, ${ }^{\mathrm{c}}$ Mark J. Krasna, MD, ${ }^{\mathrm{d}}$ Quynh-Thu Le, MD, PhD, ${ }^{e}$ Mohan Suntharalingam, MD, ${ }^{f}$ Billy W. Loo, Jr, MD, PhD, ${ }^{e}$ Chen Hu, PhD, ${ }^{b, g}$ and Jeffrey D. Bradley, MD, ${ }^{\mathrm{h}}$ for the NRG Oncology Lung Group

\section{ABSTRACT}

Objective: Concern exists regarding surgery after thoracic radiation. We aimed to assess early results of anatomic resection following induction therapy with platinum-based chemotherapy and full-dose thoracic radiation for resectable $\mathrm{N} 2+$ stage IIIA non-small cell lung cancer.

Methods: Two prospective trials were recently conducted by NRG Oncology in patients with resectable $\mathrm{N} 2+$ stage IIIA non-small cell lung cancer with the primary end point of mediastinal node sterilization following concurrent full-dose chemoradiotherapy (Radiation Therapy Oncology Group trials 0229 and 0839). All surgeons demonstrated postinduction resection expertise. Induction consisted of weekly carboplatin (area under the curve, 2.0) and paclitaxel $\left(50 \mathrm{mg} / \mathrm{m}^{2}\right)$ and concurrent thoracic radiation $60 \mathrm{~Gy}(0839) / 61.2 \mathrm{~Gy}$ (0229) in 30 fractions. Patients in study 0839 were randomized 2:1 to weekly panitumumab + chemoradiotherapy or chemoradiotherapy alone during induction. Primary results were similar in all treatment arms and reported previously. Short-term surgical outcomes are reported here.

Results: One hundred twenty-six patients enrolled; 93 (74\%) had anatomic resection, 77 underwent lobectomy, and 16 underwent extended resection. Microscopically margin-negative resections occurred in $85(91 \%)$. Fourteen $(15 \%)$ resections were attempted minimally invasively, including 2 converted without event. Grade 3 or 4 surgical adverse events were reported in $26(28 \%)$, 30-day mortality in $4(4 \%)$ and 9o-day mortality in $5(5 \%)$. Patients undergoing extended resection experienced similar rates of grade 3 or 4 adverse events (odds ratio, $0.95 ; 95 \%$ confidence interval, $0.42-3.8$ ) but higher 30 -day (1.3\% vs $18.8 \%$ ) (odds ratio, $17.54 ; 95 \%$ confidence interval, $1.75-181.8$ ) and 90-day mortality (2.6\% vs $18.8 \%$ ) (odds ratio, 8.65 ; $95 \%$ confidence interval, 1.3-56.9).

Conclusions: Lobectomy was performed safely following full-dose concurrent chemoradiotherapy in these multi-institutional prospective trials; however, increased mortality was noted with extended resections. ( $J$ Thorac Cardiovasc Surg 2020;160:1331-45)

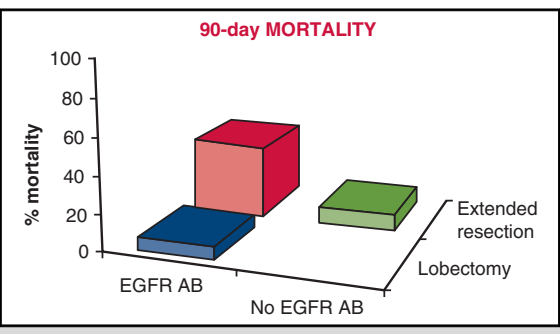

NRG Oncology 0229 and 0839 trials assessed fulldose concurrent chemoradiotherapy and resection.

\section{CENTRAL MESSAGE}

Lobectomy was performed safely following full-dose concurrent chemoradiotherapy in these multi-institutional prospective trials.

\section{PERSPECTIVE STATEMENT}

Controversy exists over ideal induction strategy for resectable N2+ stage IIIA NSCLC. Full-dose concurrent chemoradiotherapy results in high rates of mediastinal lymph node sterilization, but there is fear of operating after full-dose radiation. This multi-institutional prospective analysis suggests no excess in morbidity or mortality for lobectomy following full-dose concurrent chemoradiotherapy.

See Commentaries on pages 1346 and 1348.
From the ${ }^{\mathrm{a}}$ Department of Surgery, University of Chicago Medicine and Biologic Sciences, Chicago, Ill; ${ }^{\mathrm{b}} \mathrm{NRG}$ Oncology Statistics and Data Management Center, Philadelphia, Pa; ${ }^{\mathrm{c} D i v i s i o n}$ of Medical Oncology, Department of Medicine,University of Maryland Medical Center, Baltimore, Md; ${ }^{\mathrm{d}}$ Department of Surgery, Jersey Shore University Medical Center, Neptune City, NJ; ${ }^{\mathrm{e}}$ Department of Radiation Oncology, Stanford Cancer Institute, Stanford, Calif; ${ }^{\mathrm{f}}$ Department of Radiation Oncology, University of Maryland School of Medicine, Baltimore, Md; ${ }^{\mathrm{g}}$ Division of Biostatistics and Bioinformatics, Sidney Kimmel Comprehensive Cancer Center, Johns Hopkins University School of Medicine, Baltimore, Md; and ${ }^{\mathrm{h}}$ Department of Radiation Oncology, Winship Cancer Institute, Emory University, Atlanta, Ga.

Supported by grants U10CA180868 (NRG Oncology Operations), U10CA180822 (NRG Oncology Statistics and Data Management Center), and U24CA180803
(Imaging and Radiation Oncology Core) from the National Cancer Institute and Amgen.

Dr Edelman is currently with Fox Chase Cancer Center, Philadelphia, Pa.

Read at the 97th Annual Meeting of The American Association for Thoracic Surgery, Boston, Massachusetts, April 29-May 3, 2017.

Received for publication May 11, 2017; revisions received March 26, 2020; accepted for publication March 26, 2020; available ahead of print May 31, 2020.

Address for reprints: Jessica S. Donington, MD, Department of Thoracic Surgery, 60637 (E-mail: jdonington@uchicago.edu).

$0022-5223 / \$ 36.00$

Copyright (C) 2020 by The American Association for Thoracic Surgery https://doi.org/10.1016/j.jtcvs.2020.03.171 


$$
\begin{aligned}
& \text { Abbreviations and Acronyms } \\
& \begin{aligned}
\text { AE } & =\text { adverse events } \\
\text { CRT } & =\text { chemoradiotherapy } \\
\text { EGFR } & =\text { epidermal growth factor receptor } \\
\text { GTV } & \text { gross tumor volume } \\
\text { LN } & =\text { lymph node } \\
\text { NSCLC } & =\text { non-small cell lung cancer } \\
\text { OS } & =\text { overall survival } \\
\text { RT } & =\text { radiation therapy } \\
\text { R0 } & =\text { microscopically margin-negative } \\
& \text { resection } \\
\text { RTOG }= & \text { Radiation Therapy Oncology Group } \\
\text { STS } & \text { Society of Thoracic Surgeons } \\
\text { GTSDB }= & \text { General Thoracic Surgery Database }
\end{aligned}
\end{aligned}
$$

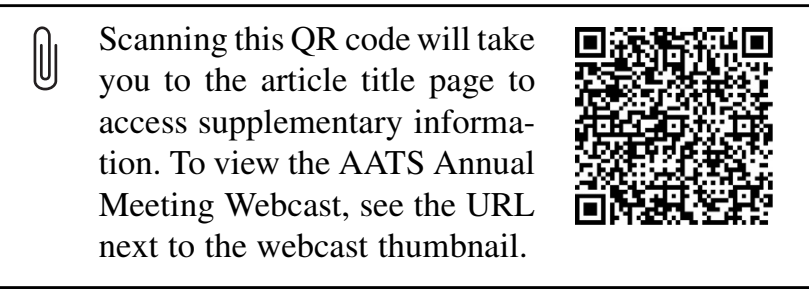

Stage III non-small cell lung cancer (NSCLC) presents a treatment challenge. Patients are treated for cure, but only $20 \%$ to $30 \%$ achieve that goal, and acceptable treatment plans vary dramatically. ${ }^{1}$ Chemotherapy-based multimodality therapy is standard of care, but ideal local therapy is unclear. Concurrent chemoradiotherapy (CRT) without surgery is commonly accepted as the standard of care, ${ }^{2}$ but local relapse is the first site of failure in $>30 \%{ }^{3}$ Therefore, adding surgery to improve outcomes is attractive. Lung resection following induction CRT to 45 Gy was shown to be safe in Southwest Oncology Group $8805^{4}$ and the Pancoast Intergroup study $0160 .^{5}$ The Intergroup trial 0139 evaluated adding surgery to CRT for the treatment for N2+ IIIA NSCLC. ${ }^{6}$ The primary end point was improved overall survival (OS), but was not met despite a significant improvement in progression-free survival. Some of this discrepancy was due to an unacceptably high number of patients undergoing pneumonectomy and excessive mortality following pneumonectomy. ${ }^{6}$ Preoperative radiotherapy (RT) was limited to $45 \mathrm{~Gy}$ in these trials, but multiple single institution series report the safety of lung resection following CRT to $60 \mathrm{~Gy} .{ }^{7,8} \mathrm{NRG}$ Oncology Radiation Therapy Oncology Group (RTOG) 0229 trial (0229) and RTOG 0839 trial (0839) evaluated the feasibility of a multicenter trial of trimodality therapy in which surgical resection followed CRT with full-dose (60 Gy) RT. Surgical certification was required in both trials to ensure expertise in postinduction lung resections (Figure 1). Mediastinal lymph node (LN) sterilization was the primary end point of both trials; as a potential surrogate for long-term benefit because of its strong association with OS following induction therapy. ${ }^{6}$ RTOG 0839 (0839) also tested the hypothesis that adding an epidermal growth factor receptor (EGFR) antibody to concurrent induction CRT could improve mediastinal LN sterilization and outcomes in operable N2+ IIIA NSCLC. EGFR antibodies potentiate radiation effects in head and neck cancers, ${ }^{9}$ and RTOG trial 0324 combined cetuximab with CRT in inoperable stage III NSCLC patients and demonstrated excellent 2-year OS with minimal toxicity increase. ${ }^{10,11}$ Panitumumab is a fully human monoclonal EGFR antibody.

Primary end points for each trial were previously reported and similar in all treatment arms, mediastinal LN sterilization rate was $63 \%$ in $0229,{ }^{12} 68 \%$ in the control arm of 0839 , and $50 \%$ in the experimental arm. ${ }^{13}$ Because inclusion criteria, induction strategies, and surgical requirements were similar in the trials, short-term surgical results are combined to increase power and reported here. The primary goal of this analysis examination of short-term surgical outcome following full-dose concurrent CRT induction and determine factors associated with grade 3 or 4 adverse events (Gr3/4AEs), and 30-day and 90-day mortality.

\section{MATERIALS AND METHODS \\ Patients}

Patients with histologically documented NSCLC, stage III N2+, Eastern Cooperative Oncology Group performance status of 0 to 1 , and normal organ function (creatinine $<1.5 \mu \mathrm{L} / \mathrm{dL}$ and normal liver function tests) were potentially eligible. Mediastinal LN involvement had to be proven pathologically, and with the goal of reducing extended resections $\mathrm{LN}$ had to be $<3 \mathrm{~cm}$ in greatest diameter, and could not be in direct continuation with the primary tumor. Multimodality evaluation with the operating surgeon was required before enrollment to determine suitability for trimodality therapy and resectability. Each institution was required to have local institutional review board approval, and patients to provide written informed consent. This analysis is limited to those patients who completed CRT and anatomic resection.

\section{Induction and Consolidative Therapy}

In both trials, chemotherapy consisted of weekly carboplatin (area under the curve, 2.0$)$, paclitaxel $\left(50 \mathrm{mg} / \mathrm{m}^{2}\right)$ for 6 weeks. Weekly panitumu$\mathrm{mab}$ at $2.5 \mathrm{mg} / \mathrm{kg}$ was added during CRT in the experimental arm of 0839 . Concurrent thoracic RT in 0229 was 61.2 Gy in 1.8 -Gy daily fractions (50.4 Gy to the gross disease, ipsilateral hilar, and mediastinal nodes, and $10.8 \mathrm{~Gy}$ boost to gross disease and involved nodes). In the 0839 trial, a total of 60 Gy was delivered in 2-Gy daily fractions to gross disease only without irradiation of clinically uninvolved LNs. Consolidation chemotherapy in both trials consisted of carboplatin (area under the curve, 6) and paclitaxel $\left(200 \mathrm{mg} / \mathrm{m}^{2}\right)$ every 21 days for 2 courses.

\section{Surgery}

All surgeons required authorization from RTOG. Surgeons were all board certified in thoracic surgery, and needed to be familiar with the American Thoracic Society LN map, adhere to intraoperative requirements 


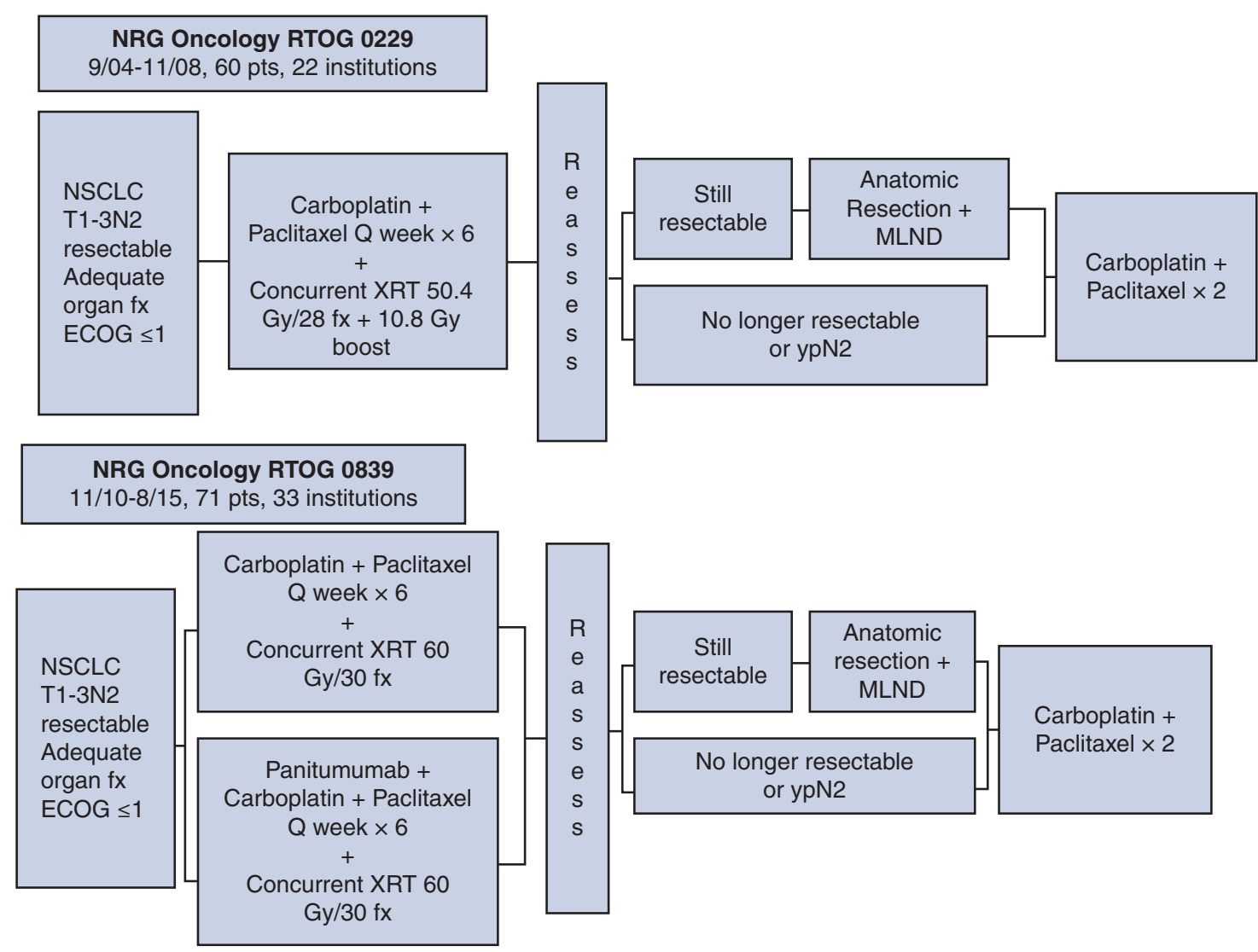

FIGURE 1. Schema for NRG Oncology trial 0229 (A) and NRG Oncology trial 0839 (B). Both were prospective, multi-institutional phase II trials evaluating the efficacy of concurrent neoadjuvant chemotherapy and high-dose chest radiation prior to resection for stage III non-small cell lung cancer (NSCLC), with the primary end point of mediastinal nodal clearance. Randomization in NRG Oncology 0839 was 2:1 in favor of research arm. RTOG, Radiation Therapy Oncology Group; ECOG, Eastern Cooperative Oncology Group; XRT, radiation therapy; MLND, mediastinal lymph node dissection.

for mediastinal staging and bronchial buttressing, and perform a minimal of 10 lobectomies or pneumonectomies per year, with 5 after induction therapy. Required presurgical evaluation following CRT included repeat history and physical, pulmonary function tests, and restaging positron emission tomography-computed tomography scans to identify changes in functional status and rule out disease progression. The mediastinum was pathologically reassessed preoperatively in a separate procedure or at the time of resection at each institution's discretion. Resections were performed 4 to 8 weeks following CRT by lobectomy or greater to achieve complete resection. Minimally invasive resections were permitted in the 0839 trial. Systematic mediastinal $\mathrm{LN}$ evaluation was required at resection, including stations $2 \mathrm{R}, 4 \mathrm{R}, 7 \mathrm{R}, 9 \mathrm{R}$, and $10 \mathrm{R}$ for right sided tumors and stations $5 \mathrm{~L}, 6 \mathrm{~L}, 7 \mathrm{~L}, 9 \mathrm{~L}$, and $10 \mathrm{~L}$ for the left side. If persistently positive mediastinal LNs were found at preoperative staging or thoracotomy, resection of the primary was at the surgeon's discretion. Bronchial stump buttressing with autologous vascularized soft-tissue was required. Patients were preferentially extubated in the operating room and postoperative fluids kept to a minimum. Major surgical deviations included resection of patients with forced expiratory volume in 1 second $<800 \mathrm{cc}$, inadequate LN sampling, lack of bronchial stump coverage, surgery $>10$ weeks after CRT, and surgery deferred for other than medical reasons. Surgical quality assurance was performed using operative notes and pathology reports at the midpoint of 0229 and continuously during 0839 . AEs were recorded by each institution per the National Cancer Institute Common Terminology Criteria for Adverse Events version 3 in 0229 and version 4 in 0839. No significant differences exist between versions for AEs reported here.

\section{Dosimetric Assessment}

Correlations between induction radiation therapy (RT) dose to specific cardiothoracic structures and short-term surgical outcomes was evaluated in the 0839 cohort (0229 radiation plans were not accessible). The lungs were delineated using automatic thresholding and excluding gross tumor volume (GTV). The heart was contoured from base to apex beginning at the origin of the ascending aorta. Dose volume histograms were generated for heart, combined lungs, ipsilateral and contralateral lungs, and esophagus.

\section{Statistical Considerations}

Univariate logistic regression methods were used to differentiate among grades 3 to 5 and less serious morbidity. When logistic regression modeling was not possible due to insufficient events $(<10$ events), Fisher exact testing was used to compare categorical outcomes and Wilcoxon rank sum was used for continuous data comparisons. For multiplicity adjustment, the Benjamini-Hochberg method was used to control the false discovery rate $<0.25$ for univariate analysis of morbidity. The sponsors were not involved in the data interpretation.

\section{RESULTS}

The trials were open sequentially, 0229 from September 2004 to November 2008 and enrolled 60 patients from 22 institutions. Trial 0839 was open November 2011 to August 
2015; it accrued 71 patients from 33 institutions before closure by the Data Monitoring Committee after a planned interim analysis. A total of $93(74.4 \%)$ patients underwent anatomic resection. Reasons for not undergoing resection were similar between trials and are outlined in (Figure 2).

The distribution of patient and tumor characteristics is presented in Table 1 . The median age was 60 years, $57 \%$ of the patients were men, and the majority were white $(85 \%)$. Most patients had T1 $(37 \%)$ or T2 $(51 \%)$ tumors. All patients had at least $1 \mathrm{~N} 2+$ node either clinically or pathologically. More tumors were situated in the right upper lobe $(47 \%)$ and were adenocarcinomas $(58 \%)$. The median forced expiratory volume in 1 second at enrollment was $2.34 \mathrm{~L}$. Only $25 \%$ of patients undergoing resection had a pathologic complete response to CRT, but $72 \%$ achieved mediastinal LN clearance.

Distribution of surgical data is presented in Table 2. The median time from the end of CRT to surgery was 42 days. Most patients had lobectomies $(83 \%)$, but lobectomy rate was higher in $0839(87 \%)$ than in $0229(77 \%)$. In 0839 , 14 resections were attempted minimally invasively, 2 converted to open. Microscopically margin-negative resections
(R0) occurred in $91 \%$ of surgical patients and $68 \%$ of all eligible patients. Median duration of surgery was 240 minutes. The number of mediastinal and hilar LN stations sampled was similar in both studies, with a median of 3 mediastinal and 2 hilar. Bronchial stump coverage was used in $84 \%$ of surgeries, most frequently with an intercostal muscle $(71 \%)$. Estimated blood loss was also lower in the 0839 cohort, and fewer patients required a transfusion than in the 0229 cohort ( $4 \%$ vs $21 \%$ ). The median length of stay was 7 days for the 0229 cohort, 5 days for the 0839 cohort.

The distribution of dosimetric data obtained from 0839 is in Table E1. The median lung $\mathrm{V}_{5}$ was $56.5 \%$, indicating that more than half of the lung excluding GTV received at least $5 \mathrm{~Gy}$ in half of the patients. The median mean dose to the lungs excluding GTV was $16.1 \mathrm{~Gy}$. Ipsilateral lung $\mathrm{V}_{5}$ was $68.2 \%$ and contralateral lung $\mathrm{V}_{5}$ was $44.3 \%$. The ipsilateral and contralateral mean lung doses were $24.5 \mathrm{~Gy}$ and $6.15 \mathrm{~Gy}$, respectively. Heart $\mathrm{V}_{10}$ was $25.3 \%$, but the median heart $\mathrm{V}_{60}$ was low at $0.7 \%$. The mean and maximum esophageal doses were $23.3 \mathrm{~Gy}$ and $63.1 \mathrm{~Gy}$.

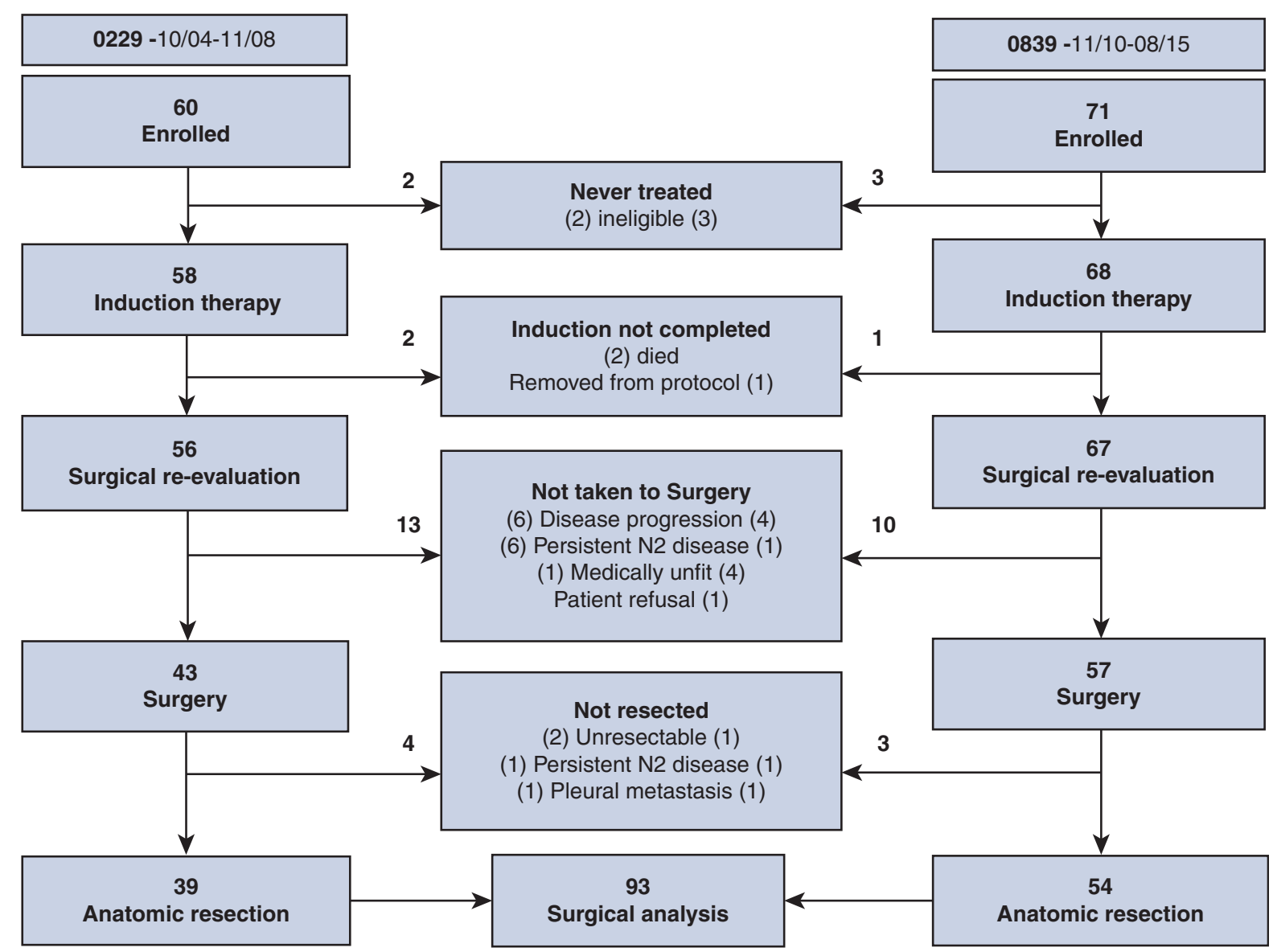

FIGURE 2. Consolidated Standards of Reporting Trials diagram for the inclusion of patients in the pooled analysis of short-term surgical outcomes form NRG Oncology trial 0229 and NRG Oncology trial 0839. Surgical outcomes analysis was limited to the 93 patients who underwent anatomic resection. 
TABLE 1. Patient and tumor characteristics

\begin{tabular}{|c|c|c|c|}
\hline Characteristic & Trial $0229(n=39)$ & Trial $0839(n=54)$ & Total $(\mathbf{N}=93)$ \\
\hline \multicolumn{4}{|l|}{ Age (y) } \\
\hline Median & 58 & 60.5 & 60 \\
\hline Min-Max & $41-75$ & $32-78$ & $32-78$ \\
\hline Q1-Q3 & $53-64$ & $54-67$ & $53-65$ \\
\hline \multicolumn{4}{|l|}{ Gender } \\
\hline Male & $23(59)$ & $30(56)$ & $53(57)$ \\
\hline Female & $16(41)$ & $24(44)$ & $40(43)$ \\
\hline \multicolumn{4}{|l|}{ Race } \\
\hline White & $30(77)$ & $49(91)$ & $79(85)$ \\
\hline Nonwhite & $9(23)$ & $5(9)$ & $14(15)$ \\
\hline \multicolumn{4}{|l|}{ T-stage at enrollment } \\
\hline $\mathrm{T} 1$ & $14(36)$ & $20(37)$ & $34(37)$ \\
\hline $\mathrm{T} 2$ & $19(49)$ & $28(52)$ & $47(50)$ \\
\hline $\mathrm{T} 3$ & $6(15)$ & $6(11)$ & $12(13)$ \\
\hline \multicolumn{4}{|l|}{ Smoking history } \\
\hline Never & $2(5)$ & $4(7)$ & $6(6)$ \\
\hline Former & $25(64)$ & $39(72)$ & $64(69)$ \\
\hline Current & $8(21)$ & $8(15)$ & $16(17)$ \\
\hline Unknown & $4(10)$ & $3(6)$ & $7(8)$ \\
\hline \multicolumn{4}{|l|}{ Pack years* } \\
\hline Median & 39 & 34 & 35 \\
\hline Min-max & $0-110$ & $0-136$ & $0-136$ \\
\hline Q1-Q3 & $30-60$ & $8.5-53$ & $15-54$ \\
\hline \multicolumn{4}{|l|}{ ECOG performance status } \\
\hline 0 & $31(84)$ & $42(78)$ & $73(80)$ \\
\hline 1 & $6(16)$ & $12(22)$ & $18(20)$ \\
\hline \multicolumn{4}{|c|}{ Clinically or pathologically $+\mathrm{N} 2$ nodes at diagnosis } \\
\hline Median & 1 & 1 & 1 \\
\hline Min-Max & $1-4$ & $1-4$ & $1-4$ \\
\hline Q1-Q3 & $1-2$ & $1-2$ & $1-2$ \\
\hline \multicolumn{4}{|l|}{ Tumor location } \\
\hline RUL & $18(46)$ & $26(48)$ & $44(47)$ \\
\hline RML & $4(10)$ & $4(7)$ & $8(9)$ \\
\hline RLL & $6(15)$ & $11(20)$ & $17(18)$ \\
\hline LUL & $7(18)$ & $11(20)$ & $18(19)$ \\
\hline LLL & $4(10)$ & $2(4)$ & $6(6)$ \\
\hline \multicolumn{4}{|l|}{ Histology } \\
\hline Adenocarcinoma & $19(49)$ & $35(65)$ & $54(58)$ \\
\hline Squamous & $7(18)$ & $13(24)$ & $20(21)$ \\
\hline Other & $13(33)$ & $6(11)$ & $19(20)$ \\
\hline \multicolumn{4}{|l|}{ FEV1 at baseline } \\
\hline Median & 2.3 & 2.385 & 2.34 \\
\hline Min-Max & $1.28-4.13$ & $1.09-3.82$ & $1.09-4.13$ \\
\hline Q1-Q3 & $1.98-2.93$ & $2.06-2.91$ & $2.06-2.92$ \\
\hline \multicolumn{4}{|l|}{ Institution type } \\
\hline Community & $12(31)$ & $15(28)$ & $27(29)$ \\
\hline Academic & $25(64)$ & $34(63)$ & $59(63)$ \\
\hline VA & $2(5)$ & $5(9)$ & $7(8)$ \\
\hline \multicolumn{4}{|l|}{ Panitumumab } \\
\hline No & 39 (100) & $19(35)$ & $58(62)$ \\
\hline Yes & $0(0.0)$ & $35(65)$ & $35(38)$ \\
\hline
\end{tabular}


TABLE 1. Continued

\begin{tabular}{|c|c|c|c|}
\hline Characteristic & Trial $0229(n=39)$ & Trial $0839(n=54)$ & Total $(\mathbf{N}=\mathbf{9 3})$ \\
\hline \multicolumn{4}{|l|}{$\mathrm{pCR}$} \\
\hline No & $29(74)$ & $41(76)$ & $70(75)$ \\
\hline Yes & $10(26)$ & $13(24)$ & $23(25)$ \\
\hline \multicolumn{4}{|l|}{ Mediastinal nodal clearance } \\
\hline No & $9(23)$ & $17(31)$ & $26(28)$ \\
\hline Yes & $30(77)$ & 37 (69) & $67(72)$ \\
\hline
\end{tabular}

\section{TABLE 2. Surgical information}

\begin{tabular}{|c|c|c|c|}
\hline Variable & Trial $0229(n=39)$ & Trial $0839(n=54)$ & Total $(\mathbf{N}=93)$ \\
\hline \multicolumn{4}{|c|}{ Days from end of radiation therapy to surgery } \\
\hline Median & 47 & 41.5 & 42 \\
\hline Min-Max & $30-78$ & $27-119$ & $27-119$ \\
\hline Q1-Q3 & $42-52$ & $38-48$ & $38-50$ \\
\hline \multicolumn{4}{|l|}{ Extent of resection } \\
\hline Lobectomy & $30(77)$ & $47(87)$ & $77(83)$ \\
\hline Pneumonectomy & $5(13)$ & $3(6)$ & $8(9)$ \\
\hline Bilobectomy & $3(8)$ & $3(6)$ & $6(6)$ \\
\hline Sleeve & $1(3)$ & $1(2)$ & $2(2)$ \\
\hline \multicolumn{4}{|l|}{ Approach } \\
\hline Open & $38(97)$ & $41(76)$ & $79(85)$ \\
\hline Minimally invasive & $1(3)$ & $13(24)$ & $14(15)$ \\
\hline \multicolumn{4}{|l|}{ R0 } \\
\hline No & $5(13)$ & $3(6)$ & $8(9)$ \\
\hline Yes & $34(87)$ & $51(94)$ & $85(91)$ \\
\hline \multicolumn{4}{|l|}{ Surgery duration (min) } \\
\hline Median & 236.5 & 246 & 240 \\
\hline Min-Max & $69-570$ & $120-2503$ & $69-2503$ \\
\hline Q1-Q3 & $199-292$ & $184-321$ & $189-318$ \\
\hline \multicolumn{4}{|c|}{ No. of mediastinal node stations sampled } \\
\hline Median & 3 & 3 & 3 \\
\hline Min-Max & $0-7$ & $1-7$ & $0-7$ \\
\hline Q1-Q3 & $2-5$ & $3-4$ & $2-4$ \\
\hline \multicolumn{4}{|c|}{ No. of hilar node stations sampled } \\
\hline Median & 2 & 2 & 2 \\
\hline Min-Max & $0-5$ & $0-5$ & $0-5$ \\
\hline Q1-Q3 & $1-3$ & $1-3$ & $1-3$ \\
\hline \multicolumn{4}{|l|}{ Bronchial coverage } \\
\hline No & $7(18)$ & $8(15)$ & $15(16)$ \\
\hline Intercostal & $26(67)$ & $40(74)$ & $66(71)$ \\
\hline Other & $6(15)$ & $4(7)$ & $10(11)$ \\
\hline \multicolumn{4}{|l|}{ Intraoperative transfusion } \\
\hline No & $31(79)$ & $52(96)$ & $83(89)$ \\
\hline Yes & $8(21)$ & $2(4)$ & $10(11)$ \\
\hline \multicolumn{4}{|l|}{ Estimated blood loss (cc) } \\
\hline Median & 200 & 150 & 175 \\
\hline Min-Max & $100-1400$ & $0-2000$ & $0-2000$ \\
\hline Q1-Q3 & $150-350$ & $62.5-259.5$ & $100-300$ \\
\hline \multicolumn{4}{|l|}{ Length of stay (d) } \\
\hline Median & 7 & 5 & 5 \\
\hline Min-Max & $2-60$ & $2-29$ & $2-60$ \\
\hline Q1-Q3 & $5-9$ & $4-7$ & $4-8$ \\
\hline
\end{tabular}

Values are presented as n (\%) unless otherwise noted. $Q 1$, First quartile; $Q 3$, third quartile; $R 0$, microscopically negative resection margin.

1336 The Journal of Thoracic and Cardiovascular Surgery • November 2020 
Nonfatal postoperative AEs were reported in 50 (53\%) patients and rates were similar between trials. Most common AEs were grade 1 to 2 atrial fibrillation $(n=20)$, atelectasis $(\mathrm{n}=18)$, and pneumothorax $(\mathrm{n}=17)$. All AEs are outlined in Table 3. Gr3/4AEs occurred 21 (23.6\%). Postoperative 30-day mortality occurred in $4(4 \%)$ and 90 -day mortality in $5(5 \%)$. For all resections the rates for any $\mathrm{AE}, \mathrm{Gr} 3 / 4 \mathrm{AE}, 30$-day mortality, and 90-day mortality were $59 \%, 28 \%, 4 \%$, and $5 \%$; for the 77 lobectomy patients the rates were $57 \%, 26 \%, 1.3 \%$, and $2.6 \%$. In a comparison of outcomes between patients who received panitumumab or not, rates of any $\mathrm{AE}(40 \%$ vs $71 \%$; $P=.003$ ) and $\mathrm{Gr} 3 / 4 \mathrm{AE}(9 \%$ vs $31 \% ; P=.02$ ) were lower with the EGFR antibody, but there was trend to higher 30 -day $(9 \%$ vs $2 \% ; P=.15)$ and 90 -day mortality $(11 \%$ vs $2 \% ; P=.06)$. The only death in the cohort that did not receive panitumumab, was a patient with postoperative pulmonary edema following pneumonectomy.

The distribution and univariate analysis for morbidity are outlined in Table 4. Panitumumab and higher T-stage were associated with decreased risk of morbidity in the full cohort, whereas extended resections were associated with an increased risk of fatal morbidity. No RT dose to heart or lung was associated with Gr3/4AEs in these models, but there was a trend toward increased risk of fatal AEs with higher lung $\mathrm{V}_{5}$, ipsilateral lung $\mathrm{V}_{10}$, and contralateral lung $V_{5}$. Details for each of mortality are outline in Table 3 and distribution for 90-day mortality is outlined in Table 5. Use of panitumumab and extended resections were associated with an increased risk for operative mortality. Although there was a trend toward increased 90-day mortality with higher ipsilateral and contralateral lung $\mathrm{V}_{5}$ and higher total lung $\mathrm{V}_{10}$, that effect was lost after taking into account panitumumab and extent of resection. These models should be viewed with caution given the low number of events.

\section{DISCUSSION}

The major finding of this analysis is that lobectomy following full-dose concurrent CRT to 60 Gy was performed safely in a multidisciplinary setting. The addition of induction therapy for patients with N2+ IIIA NSCLC who undergo resection has been standard of care since the early 1990s when a series of small phase III trials showed dramatic survival improvements compared with surgery alone. ${ }^{14,15}$ Controversy has existed ever since as to the

TABLE 3. Adverse events entire cohort $(\mathbf{N}=93)$

\begin{tabular}{|c|c|c|c|}
\hline \multicolumn{2}{|l|}{ Grade 1 or 2 AEs } & \multicolumn{2}{|l|}{ Grade 3 or 4 AEs } \\
\hline $\mathbf{A E}$ & $\bar{n}$ & $\mathbf{A E}$ & $\overline{\mathbf{n}}$ \\
\hline Atrial fibrillation & 20 & Pneumonitis & 5 \\
\hline Atelectasis & 18 & Pneumonia & 4 \\
\hline Pneumothorax & 17 & Respiratory failure & 3 \\
\hline Effusion & 16 & RLN injury & 3 \\
\hline Pain & 5 & Dyspnea & 3 \\
\hline Pneumonia & 4 & Pulmonary edema & 3 \\
\hline Chylothorax & 3 & Atelectasis & 2 \\
\hline Hypotension & 3 & Pulmonary embolus & 2 \\
\hline Hypocalcemia & 3 & $\begin{array}{l}\text { AKI, anemia, aspiration, bronchial obstruction, chylothorax, DTs, effusion, } \\
\text { empyema, esophagitis, hemorrhage, hypocalcemia, hyponatremia, } \\
\text { hypotension, hypoxia, lymphopenia, necrotic muscle flap, sepsis, } \\
\text { ventricular arrhythmia, UTI, wound infection }\end{array}$ & 1 \\
\hline Rash & 2 & & \\
\hline Constipation & 2 & & \\
\hline Anemia & 2 & & \\
\hline $\begin{array}{l}\text { ACS, arterial injury, atrial flutter, confusion, diarrhea, } \\
\text { esophagitis, hypoglycemia, ileus, narcotic OD }\end{array}$ & 1 & & \\
\hline
\end{tabular}

\begin{tabular}{lllll}
\hline & & Grade 5 AEs & & \\
\hline Demographic & \multicolumn{1}{c}{ Resection } & Panitumumab & Gr 5AE & POD \\
\hline $43 \mathrm{M}$ & R pneumonectomy & No & PPE & 6 \\
$52 \mathrm{M}$ & Bilobectomy & Yes & Hemorrhage & 23 \\
$61 \mathrm{M}$ & Lobectomy & Yes & BPF & 28 \\
$63 \mathrm{~F}$ & L pneumonectomy & Yes & Aspiration & 29 \\
$71 \mathrm{~F}$ & Lobectomy & Yes & Pneumonitis & 45 \\
\hline
\end{tabular}

Events were reported by each institution per National Cancer Institute common terminology criteria for adverse events version 3 in 0229 and version 4 in 0839 . AE, Adverse events; $R L N$, recurrent laryngeal nerve; $A K I$, acute kidney injury; DTs, delirium tremors; UTI, urinary tract infection; $A C S$, acute coronary syndrome; $O D$, overdose; $P O D$, postopertative day; $M$, male; $F$, female; $R$, right; $L$, left; $P P E$, post-pneumonectomy pulmonary edema; $B P F$, bronchopleural fistula. 
TABLE 4. Distribution of surgical morbidity and univariate models of grade $3+$ morbidity

\begin{tabular}{|c|c|c|c|c|c|c|}
\hline Variable & $\begin{array}{c}\text { Grade 3-4 } \\
(\mathrm{n}=21)\end{array}$ & $\begin{array}{c}\text { Grade } 5 \\
(n=5)\end{array}$ & $\begin{array}{c}<\text { Grade } 3 \\
\text { morbidity } \\
(n=67)\end{array}$ & $\begin{array}{c}\text { Grade } 3+ \\
\text { morbidity } \\
(\mathbf{n}=\mathbf{2 6})\end{array}$ & $\begin{array}{c}\text { Odds ratio* } \\
(95 \% \text { confidence interval })\end{array}$ & $\begin{array}{c}\text { FDR-adjusted } \\
P \text { value } \dagger\end{array}$ \\
\hline \multicolumn{7}{|l|}{ Study } \\
\hline 0229 (RL) & $10(26)$ & $1(2)$ & $28(72)$ & $11(28)$ & & \\
\hline 0839 & $11(20)$ & $4(7)$ & $39(72)$ & $15(28)$ & $0.98(0.39-2.45)$ & .96 \\
\hline \multicolumn{7}{|l|}{ Age $(y)$} \\
\hline Median & 62 & 61 & 60 & 61.5 & $1.01(0.96-1.07)$ & .64 \\
\hline Min-Max & $41-72$ & $44-71$ & $32-78$ & $41-72$ & & \\
\hline Q1-Q3 & $56-65$ & $52-69$ & $53-65$ & $54-66$ & & \\
\hline \multicolumn{7}{|l|}{ Gender } \\
\hline Male (RL) & $9(17)$ & $3(6)$ & $41(77)$ & $12(23)$ & & \\
\hline Female & $12(30)$ & $2(5)$ & $26(65)$ & $14(35)$ & $1.84(0.74-4.59)$ & .33 \\
\hline \multicolumn{7}{|l|}{ Race } \\
\hline White (RL) & $19(24)$ & $5(6)$ & $55(70)$ & $24(30)$ & & \\
\hline Nonwhite & $2(14)$ & $0(0)$ & $12(86)$ & $2(14)$ & $0.38(0.08-1.84)$ & .33 \\
\hline \multicolumn{7}{|l|}{ T-Stage } \\
\hline $\mathrm{T} 1$ (RL) & $12(3)$ & $3(9)$ & $19(56)$ & $15(44)$ & & \\
\hline $\mathrm{T} 2 / \mathrm{T} 3$ & $9(15)$ & $2(3)$ & $48(81)$ & $11(19)$ & $0.29(0.11-0.74)$ & .17 \\
\hline \multicolumn{7}{|l|}{$\mathrm{cN} 2$ positive stations } \\
\hline $1(\mathrm{RL})$ & $16(26)$ & $4(7)$ & $41(67)$ & $20(33)$ & & \\
\hline $2-4$ & $5(16)$ & $1(3)$ & $26(81)$ & $6(19)$ & $0.47(0.17-1.33)$ & .33 \\
\hline \multicolumn{7}{|l|}{ Tumor location, right vs left } \\
\hline Right-sided (RL) & $17(25)$ & $4(6)$ & $48(70)$ & $21(30)$ & & \\
\hline Left-sided & $4(17)$ & $1(4)$ & $19(79)$ & $5(21)$ & $0.60(0.20-1.83)$ & .48 \\
\hline \multicolumn{7}{|l|}{ Tumor location - upper vs lower } \\
\hline Upper lobe (RL) & $13(21)$ & $3(5)$ & $45(74)$ & $16(26)$ & & \\
\hline Lower/middle lobe & $8(25)$ & $2(6)$ & $22(69)$ & $10(31)$ & $1.28(0.50-3.27)$ & .61 \\
\hline \multicolumn{7}{|l|}{ Histology } \\
\hline Adenocarcinoma/other (RL) & $14(19)$ & $4(6)$ & $55(75)$ & $18(25)$ & & \\
\hline Squamous & $7(35)$ & $1(5)$ & $12(60)$ & $8(40)$ & $2.04(0.72-5.77)$ & .33 \\
\hline \multicolumn{7}{|l|}{ FEV1 } \\
\hline Median & 2.16 & 2.24 & 2.41 & 2.20 & $0.75(0.36-1.53)$ & .49 \\
\hline Min-Max & $1.28-3.82$ & $1.65-3.36$ & $1.09-4.13$ & $1.28-3.82$ & & \\
\hline Q1-Q3 & $1.97-2.85$ & $2.16-2.39$ & $2.09-2.93$ & $1.97-2.85$ & & \\
\hline \multicolumn{7}{|l|}{ Institution type } \\
\hline Community/VA (RL) & $9(26)$ & $2(6)$ & $23(68)$ & $11(32)$ & & \\
\hline Academic & $12(20)$ & $3(5)$ & $44(75)$ & $15(25)$ & $0.71(0.28-1.80)$ & .52 \\
\hline \multicolumn{7}{|l|}{ Received panitumumab } \\
\hline No (RL) & $18(31)$ & $1(2)$ & $39(67)$ & $19(33)$ & & \\
\hline Yes & $3(9)$ & $4(11)$ & $28(80)$ & $7(20)$ & $0.51(0.19-1.39)$ & .33 \\
\hline \multicolumn{7}{|l|}{$\mathrm{pCR}$} \\
\hline No (RL) & $15(21)$ & $3(4)$ & $52(74)$ & $18(26)$ & & \\
\hline Yes & $6(26)$ & $2(9)$ & $15(65)$ & $8(35)$ & $1.54(0.56-4.24)$ & .49 \\
\hline \multicolumn{7}{|l|}{ Mediastinal LN clearance } \\
\hline No (RL) & $6(23)$ & $2(8)$ & $18(69)$ & $8(31)$ & & \\
\hline Yes & $15(22)$ & $3(5)$ & $49(73)$ & $18(27)$ & $0.83(0.31-2.23)$ & .71 \\
\hline \multicolumn{7}{|l|}{ Days from end of RT to surgery } \\
\hline Median & 48 & 38 & 42 & 44 & $1.02(0.99-1.05)$ & .44 \\
\hline Min-Max & $33-119$ & $38-47$ & $27-106$ & $33-119$ & & \\
\hline Q1-Q3 & $42-54$ & $38-39$ & $38-49$ & $39-51$ & & \\
\hline
\end{tabular}


TABLE 4. Continued

\begin{tabular}{|c|c|c|c|c|c|c|}
\hline Variable & $\begin{array}{c}\text { Grade } 3-4 \\
(n=21)\end{array}$ & $\begin{array}{c}\text { Grade } 5 \\
(n=5)\end{array}$ & $\begin{array}{c}<\text { Grade } 3 \\
\text { morbidity } \\
(\mathbf{n}=67)\end{array}$ & $\begin{array}{c}\text { Grade 3+ } \\
\text { morbidity } \\
(\mathbf{n}=\mathbf{2 6})\end{array}$ & $\begin{array}{c}\text { Odds ratio } * \\
\text { 95\% confidence interval) }\end{array}$ & $\begin{array}{c}\text { FDR-adjusted } \\
P \text { value }\end{array}$ \\
\hline \multicolumn{7}{|l|}{ Extended resection } \\
\hline Lobectomy (RL) & $18(23)$ & $2(3)$ & $57(74)$ & $20(26)$ & & \\
\hline Extended resection & $3(19)$ & $3(19)$ & $10(62)$ & $6(38)$ & $1.71(0.55-5.31)$ & .48 \\
\hline \multicolumn{7}{|l|}{ Surgical approach } \\
\hline Open (RL) & $18(23)$ & $4(5)$ & $57(72)$ & $22(28)$ & & \\
\hline Minimally invasive & $3(21)$ & $1(7)$ & $10(71)$ & $4(29)$ & $1.04(0.29-3.65)$ & .96 \\
\hline \multicolumn{7}{|l|}{$\mathrm{R} 0$ resection } \\
\hline No (RL) & $0(0)$ & $0(0)$ & $8(100)$ & $0(0)$ & N/A & $.10 \ddagger$ \\
\hline Yes & $21(25)$ & $5(6)$ & $59(69)$ & $26(31)$ & & \\
\hline \multicolumn{7}{|l|}{ Surgery duration } \\
\hline Median & 243 & 305 & 233 & 248 & $1.00(1.00-1.00)$ & .94 \\
\hline Min-Max & $180-561$ & $180-397$ & $69-2503$ & $180-5641$ & & \\
\hline Q1-Q3 & 218-297 & $248-362$ & $184-312$ & $218-326$ & & \\
\hline \multicolumn{7}{|c|}{ No. of mediastinal LN sampled } \\
\hline Median & 3 & 4 & 3 & 3.5 & $1.09(0.82-1.44)$ & .58 \\
\hline Min-Max & $1-7$ & $1-7$ & $0-7$ & $1-7$ & & \\
\hline \multicolumn{7}{|l|}{ No. of hilar LN sampled } \\
\hline Median & 2 & 2 & 2 & 2 & $0.66(0.42-1.03)$ & .25 \\
\hline Min-Max & $0-4$ & $1-3$ & $0-5$ & $0-4$ & & \\
\hline \multicolumn{7}{|l|}{ Bronchial coverage } \\
\hline No (RL) & $4(24)$ & $0(0)$ & $13(76)$ & $4(24)$ & & \\
\hline Yes & $17(22)$ & $5(6)$ & $54(71)$ & $22(29)$ & $1.32(0.39-4.51)$ & .65 \\
\hline \multicolumn{7}{|c|}{ Intraoperative transfusion } \\
\hline No (RL) & $18(22)$ & $5(6)$ & $60(72)$ & $23(28)$ & & \\
\hline Yes & $3(30)$ & $0(0)$ & $7(70)$ & $3(30)$ & $1.12(0.27-4.70)$ & .88 \\
\hline \multicolumn{7}{|l|}{ Estimated blood loss } \\
\hline Median & 175 & 88 & 200 & 150 & $1.00(1.00-1.00)$ & .33 \\
\hline Min-Max & $0-500$ & $50-300$ & $10-2000$ & $0-500$ & & \\
\hline Q1-Q3 & $100-300$ & $50-213$ & $100-300$ & $87.5-275$ & & \\
\hline RTOG 0839 RT data & $\begin{array}{c}\text { Grade } 3-4 \\
(n=11) \\
\end{array}$ & $\begin{array}{c}\text { Grade } 5 \\
(n=4) \\
\end{array}$ & $\begin{array}{c}<\text { Grade } 3 \\
\text { morbidity } \\
(\mathrm{n}=\mathbf{3 9}) \\
\end{array}$ & $\begin{array}{c}\text { Grade } 3+ \\
\text { morbidity } \\
(n=15) \\
\end{array}$ & $\begin{array}{c}\text { Odds ratio } \\
(95 \% \text { confidence } \\
\text { interval }) \\
\end{array}$ & $P$ value \\
\hline \multicolumn{7}{|l|}{ Lung $\mathrm{V}_{5}$} \\
\hline Median & 55.9 & 75.0 & 52.9 & 57.7 & $1.03(1.00-1.07)$ & .25 \\
\hline Min-Max & $44.0-86.0$ & $57.7-88.2$ & $19.0-85.1$ & $44.0-63.8$ & & \\
\hline Q1-Q3 & $49.0-72.9$ & $60.8-87.1$ & $41.2-67.4$ & $34.4-50.6$ & & \\
\hline \multicolumn{7}{|l|}{ Ipsilateral lung $\mathrm{V}_{10}$} \\
\hline Median & 60.9 & 76.2 & 61.1 & 62.9 & $1.03(1.00-1.07)$ & .25 \\
\hline Min-Max & $46.1-79.6$ & $56.3-95.5$ & $26.5-87.0$ & $46.1-95.5$ & & \\
\hline Q1-Q3 & $50.7-72.2$ & $61.1-91.0$ & $44.2-72.0$ & $51.7-77.5$ & & \\
\hline \multicolumn{7}{|l|}{ Contralateral lung $\mathrm{V}_{5}$} \\
\hline Median & 45.1 & 64.5 & 42.4 & 55.6 & $1.03(1.00-1.06)$ & .25 \\
\hline Min-Max & $29.8-85.6$ & $49.6-83.0$ & $0.5-83.4$ & $29.8-85.6$ & & \\
\hline Q1-Q3 & $37.4-64.0$ & $52.6-78.2$ & $24.2-58.2$ & $39.8-67.7$ & & \\
\hline
\end{tabular}

Values are presented as n (\%) unless otherwise indicated. $F D R$, False discovery rate; $R L$, reference level; $R O$, microscopically negative resection margin; $Q 1$, first quartile; $Q 3$, third quartile; $F E V 1$, forced expiratory volume in 1 second; $V A$, Veterans Administration; $p C R$, pathologic complete response; $L N$, lymph node; $R T$, radiation therapy; $R T O G$, Radiation Therapy Oncology Group. *Odds ratio modeling the risk of developing grade $3+$ morbidity. $\dagger P<.25$ was considered significantly different. $\ddagger$ Fisher exact test. 
TABLE 5. Surgical mortality

\begin{tabular}{|c|c|c|c|c|}
\hline \multirow[b]{2}{*}{ Variable } & \multicolumn{2}{|c|}{ 30-d Mortality } & \multicolumn{2}{|c|}{ 90-d Mortality } \\
\hline & No & Yes & No & Yes \\
\hline \multicolumn{5}{|l|}{ Received panitumumab } \\
\hline No & $57(98)$ & $1(2)$ & $57(98)$ & $1(2)$ \\
\hline Yes & $32(91)$ & $3(9)$ & $31(89)$ & $4(11)$ \\
\hline$P$ value* & .15 & & .06 & \\
\hline \multicolumn{5}{|l|}{ Extended resection } \\
\hline Lobectomy & $76(99)$ & $1(1)$ & 75 (97) & $2(3)$ \\
\hline Extended resection & $13(81)$ & $3(19)$ & $13(81)$ & $3(19)$ \\
\hline$P$ value* & .02 & & .03 & \\
\hline RTOG 0839 RT Data & $\mathrm{n}=51$ & $\mathrm{n}=3 \dagger$ & $\mathrm{n}=50$ & $\mathrm{n}=4$ \\
\hline \multicolumn{5}{|l|}{ Lung $\mathrm{V}_{5}$} \\
\hline Median & 55.9 & $57.7,64.0,86.0^{\dagger}$ & 55.0 & 75.0 \\
\hline Min-Max & $19.0-88.2$ & & $19.0-86.0$ & $57.7-88.2$ \\
\hline Q1-Q3 & $46.0-69.2$ & & $46.0-68.3$ & $60.8-87.1$ \\
\hline$P$ value $\ddagger$ & 0.17 & & 0.04 & \\
\hline \multicolumn{5}{|l|}{ Ipsilateral lung $\mathrm{V}_{10}$} \\
\hline Median & 61.1 & $56.3,65.9,95.9 \dagger$ & 61.0 & 76.2 \\
\hline Min-Max & $26.5-87.0$ & & $26.5-87.0$ & $56.3-95.5$ \\
\hline Q1-Q3 & $48.0-72.2$ & & $48.0-72.0$ & $61.1-91.0$ \\
\hline$P$ value $\ddagger$ & 0.30 & & 0.09 & \\
\hline \multicolumn{5}{|l|}{ Contralateral lung $\mathrm{V}_{5}$} \\
\hline Median & 43.4 & $49.6,55.6,73.4 \dagger$ & 42.9 & 64.5 \\
\hline Min-Max & $0.5-85.6$ & & $0.5-85.6$ & $49.6-83.0$ \\
\hline Q1-Q3 & $26.7-64.0$ & & $26.7-62.9$ & $52.6-78.2$ \\
\hline$P$ value $\ddagger$ & 0.25 & & 0.07 & \\
\hline
\end{tabular}

Values are presented as n (\%) unless otherwise noted. $R T O G$, Radiation Therapy Oncology Group; $R T$, radiation therapy; $Q 1$, first quartile; $Q 3$, third quartile. *Fisher exact test. $\dagger$ All 3 values are reported due to small number of events. $\ddagger$ Wilcoxon rank sum test

best induction strategy, with considerable debate of safety and efficacy of preoperative RT. Full-dose concurrent CRT appears to provide the highest rates of mediastinal LN sterilization, ${ }^{16-18}$ but many are hesitant to use it due to high rates of operative complications reported in early series. ${ }^{4,19}$ Several single-institution studies have reported the feasibility of full-dose concurrent CRT and resection, ${ }^{7,8,20,21}$ but these 2 RTOG trials are the first to prospectively evaluate this approach in a multi-institutional setting. These findings provide an important and timely baseline as the field moves into an era where immunotherapy is being added to induction regimens.

Two recent randomized trials from Europe attempted to define the role of induction CRT in resectable N2+ NSCLC. The Clinical Trial Group for the German Cancer Society compared CRT plus resection to CRT alone, similar to the Intergroup 0139 trial, but used a more complex induction protocol, and limited enrollment to high-volume thoracic centers. ${ }^{22}$ RT in the surgery group was limited to $45 \mathrm{~Gy}$, two-thirds of patients had IIIB disease and extended resections were used in $43 \%$. Similar to the surgical arm of the Intergroup, 90-day operative mortality was 7\%.

The second trial from the Swiss Cancer Research Group randomized 232 patients to 3 cycles of cisplatin-based chemotherapy alone or with the addition of RT to $44 \mathrm{~Gy}$ in a sequential fashion followed by resection in both arms. ${ }^{23}$ The low RT dose and sequential approach limit the ability to evaluate the trimodality approach. Overall operative morbidity was $23 \%$ and mortality was $1.5 \%$, and neither were increased in the RT arm. This trial had more extended resections (34\%) and fewer R0 resections $(86 \%)$ suggesting some difference in inclusion criteria or treatment response to the data presented here. Overall, the $23 \% \mathrm{Gr} 3 / 4 \mathrm{AEs}$ and $4 \%$ 30-day mortality from the RTOG trials compares favorably to these European prospective trials.

The short-term outcomes for lobectomy in this trial also compare favorably with contemporary outcomes for NSCLC resections from the Society for Thoracic Surgery General Thoracic Surgery Database (STS-GTSDB) and resections following chemotherapy alone in stage IIIA disease. Morbidity and mortality following NSCLC resection reported in the STS-GTSDB from 2002-2008 were 18.5\% and $2.2 \%$, respectively, ${ }^{24}$ and lower between 2012 and 2014, where 30-day mortality was only $1.4 \%$ for all NSCLC resections and $1.3 \%$ for lobectomy. ${ }^{25}$ The 30-day mortality rates following lobectomy in this series are the same despite higher stage, fewer minimally invasive procedures, and full-dose induction CRT. Similar to outcomes in the STS-GTSDB, thoracic surgical expertise may explain 
the short-term outcomes of this analysis compared with previous prospective analysis of trimodality therapy, most specifically, the Intergroup trial 0139. Participating surgeons in these trials were required to be board certified in thoracic surgery, demonstrate experience with resections following induction therapy, and pretreatment surgical evaluation was required before enrollment.

An equally important finding of this analysis is that extended resections were associated with increased mortality, $19 \%$ at 30 and 90 days. Excessive mortality following pneumonectomy ${ }^{6,19}$ and bilobectomy ${ }^{26}$ as part of trimodality therapy has been previously reported. Extended resections did not portend an increased rate of Gr3/4AEs, but when an $\mathrm{AE}$ occurred, patients were significantly more likely to die as a result compared with those with AEs following lobectomy. Failure to rescue is an important concept in systems related to perioperative care; induction therapy ${ }^{27}$ and extended resections ${ }^{28}$ have independently identified as risks factors for mortality following lung resection, and the combination may expose weaknesses in the biological system. The decreased tolerance of complications increases the importance of appropriate patient selection and intraoperative skill to avoid extended resections in this setting. Excluding patients with central tumors and bulky nodes, decreased, but did not eliminate use of extended resections in these trials.

The complications related to the panitumumab in the 0839 trial limit some of the applicability of this work, but are also a meaningful finding. The addition of an EGFR antibody did not improve the primary outcome and was associated with increased mortality following resection. The etiology of this toxicity is unclear because similar toxicity is not reported with use of EGFR antibodies before resections for other histologies. ${ }^{9,29}$ It is significant to note the toxicity of EGFR antibodies with RT is related to local inflammation, ${ }^{30}$ suggesting pneumonitis as a potential cause. This increased toxicity is in line with the results from RTOG 0617, the large 4-arm phase III trial in unresectable stage III NSCLC, where neither the addition of an EGFR antibody nor the increase in RT to 74 Gy improved survival over standard concurrent CRT to $60 \mathrm{~Gy}$. ${ }^{1}$ More severe toxicity and treatment-related deaths occurred in the cetuximab arms of this trial. These results from RTOG 0617 were not yet known when 0839 was designed and implemented, but had been identified in 2015 at the time of the interim analysis which resulted in the trial's early closure. This therefore represents the second NSCLC trial to report increased toxicity with an EGFR antibody wit in combination with concurrent chemotherapy and full-dose RT.

A rarely discussed benefit of high dose concurrent CRT over other induction strategies is that concurrent CRT to $60 \mathrm{~Gy}$ is standard of care for unresectable stage IIIA NSCLC,${ }^{31}$ and a significant proportion of patients $(20.8 \%$ in this trial) who start a trimodality treatment plan, do not undergo resection due to progression or medical decline. This group is not included in our retrospective series. The reasons for not undergoing surgery in this analysis were progression outside of RT field, persistent $\mathrm{N} 2$ on preresection biopsy, and medical decline. In this approach, these patients are provided definitive therapy without delays, gaps, or sequential RT, and now have the potential for consolidative immunotherapy. The response induction treatment serves as an important in vivo test of tumor biology. These results also provide evidence for resection in patients referred with residual or persistent disease after definitive CRT, although the majority of resections in these series were all performed within 8 weeks of CRT completion and may not be applicable for resections performed after a prolonged interval.

Recent data on definitive CRT in unresectable stage III NSCLC patients indicate heart dose may be responsible to increased toxicity and poor OS. ${ }^{32,33}$ Correlations between induction RT dose to specific cardiothoracic structures (heart, esophagus, ipsilateral, and contralateral lung) and short-term surgical outcomes has not been previously investigated, but was queried here in the 0839 cohort. Although there was a trend toward increased severe toxicity and 90day mortality with increasing ipsilateral and contralateral $\mathrm{V}_{5}$ and lung $\mathrm{V}_{10}$, the number of events was small and the effect was not seen after controlling for panitumumab and extent of resection.

This analysis has several limitations. First, its small size, despite being a combined cohort from 2 prospective RTOG trials, only 93 patients were evaluable. The subsequent limited number of events (4 deaths within 30 days and 5 at 90 days) makes the toxicity models for mortality exploratory. This was further hindered by the lack of RT planning data from the 0229 trial. Although a trend toward increased toxicity was noted with increase ipsilateral and contralateral lung $\mathrm{V}_{5}$, and total lung $\mathrm{V}_{10}$, events were too few for definitive conclusions. This topic deserves greater investigation, and could provide RT planning guidance to reduce surgical morbidity without decreasing the benefit of mediastinal LN sterilization. Second, the trials permitted surgeons to defer resection in patients with persistent nodal disease on postinduction invasive staging. This design allowed for evaluation of the primary outcome of mediastinal nodal clearance, but may have skewed the population in this analysis. A third limitation is the lacks of diffusion data from pulmonary function tests, which has strong correlation with shortterm lobectomy outcomes.

Additionally, these trials employed the low dose carboplatin/paclitaxel regimen with concurrent RT. Some argue this approach results in lower chemotherapy doses and decreased efficacy outside of the RT field. The same regime was used in the control arm of RTOG 0617, and resulted in $58 \%$ OS at 2 years, the best results for unresectable stage III reported before use of immune therapies. ${ }^{1}$ Two recent 
meta-analyses also demonstrate comparable outcomes with less toxicity for this approach compared with a full-dose cisplatin-based regimen. ${ }^{34,35}$ Therefore, the low-dose carboplatin/paclitaxel regimen seems uniquely suited for the trimodality approach. It allows for delivery of full-dose induction RT, and chemotherapy in the consolidation setting.

Finally, this analysis examines only short-term surgical outcomes and does not look at survival or toxicity past 90 days. Although it is recognized that full-dose induction CRT increases rates of pathologic complete response and mediastinal LN sterilization, it is less clear if that translates to improved OS survival compared with lower doses or bimodality approaches. A major question regarding aggressive CRT induction strategies is the potential for increased long-term noncancer mortality. ${ }^{18}$ Others hypothesize that the response to systemic therapy is the driver of primary long-term survival and local control is secondary and should be achieved by whichever therapy can provide it with the least toxicity. ${ }^{17}$ Unfortunately, data from retrospective analyses are influenced by inherent treatment bias and of limited value when comparing such divergent treatments. Some of these issues could be addressed in longer-term follow-up of this cohort.

\section{CONCLUSIONS}

Tri-modality therapy with concurrent high-dose CRT remains a viable option for well-selected patients with stage IIIA NSCLC, but was associated with excessive mortality when combined with extended resections. This approach is known to increase sterilization of mediastinal LNs, which is strongly associated with OS benefit. We have demonstrated the ability of thoracic surgeons at multiple institutions, including community and Veterans Administration hospitals, to perform these resections safely in the context of a clinical trial. Additional follow-up is required to determine long-term benefit of the trimodality approach.

\section{Conflict of Interest Statement}

Dr Donington reports having received honorarium and travel expenses from AstraZeneca, outside the submitted work. Ms. Paulus reports receiving grants from Amgen for the work under consideration. Dr Edelman reports grants (to institution) and consulting fees from BMS, Lilly Oncology, Ariad, and Genentech outside the submitted work. Dr Le reports grants from RedHil, Varian, and Amgen and travel fees from BMS outside the submitted work. Dr Loo Jr reports grants from Varian Medical Systems and RaySearch Laboratories, honoraria from Varian Medical Systems, and that he is a board member of TibaRay, Inc, outside the submitted work. Dr Robinson reports having received grants from Varian and Elekta and personal fees from Varian and ViewRay outside the submitted work. Dr Diehn reports a grant from Varian Medical Systems outside the submitted work. Dr Decker reports a grant from
Merck\& Co and advisory board fees from Regeneron Pharmaceuticals outside the submitted work. Dr Hu reports nonfinancial support from Varian Medical Systems outside the submitted work. Dr Bradley reports a grant (to institution) and travel fees from Mevion Medical Systems, Inc, and scientific advisory board fees from ViewRay and Varian Medical outside the submitted work. All other authors reported no conflicts of interest.

The Journal policy requires editors and reviewers to disclose conflicts of interest and to decline handling or reviewing manuscripts for which they may have a conflict of interest. The editors and reviewers of this article have no conflicts of interest.

\section{Webcast \\ You can watch a Webcast of this AATS meeting presenta- tion by going to: https://aats.blob.core.windows.net/media/ 17AM/2017-05-01/BallroomABC/05-01-17_BallroomABC_ 1020_Donington.mp4.}

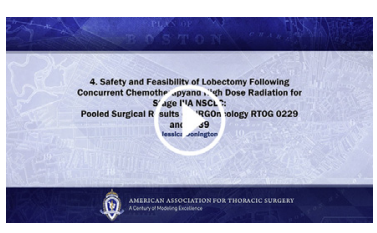

The authors thank the following doctors for accruing patients to the studies associated with this work: Steven J. Feigenberg, MD, University of Maryland School of Medicine; Elizabeth M. Gore, MD, Medical College of Wisconsin; Vita V. McCabe, MD, Michigan Cancer Research Consortiums; Cliff G. Robinson, MD, Washington University School of Medicine; Gregory M. Videtic, MD, Case Western Reserve/University Hospital Seidman Cancer Center; Nathaniel R. Evans, MD, Thomas Jefferson University; Sidney Kimmel Medical College; Paul J. Thurmes, MD, Metro-Minnesota Community Clinical Oncology Program; Maximilian Diehn, MD, PhD, Stanford Cancer Institute; and Roy H. Decker, MD, Yale School of Medicine.

\section{References}

1. Bradley JD, Paulus R, Komaki R, Masters G, Blumenschein G, Schild S, et al Standard-dose versus high-dose conformal radiotherapy with concurrent and consolidation carboplatin plus paclitaxel with or without cetuximab for patients with stage IIIA or IIIB non-small-cell lung cancer (RTOG 0617): a randomised, two-by-two factorial phase 3 study. Lancet Oncol. 2015;16:187-99.

2. Bezjak A, Temin S, Franklin G, Giaccone G, Govindan R, Johnson ML, et al. Definitive and adjuvant radiotherapy in locally advanced non-small-cell lung cancer: American Society of Clinical Oncology clinical practice guideline endorsement of the American Society for Radiation Oncology evidence-based clinical practice guideline. J Clin Oncol. 2015;33:2100-5.

3. Furuse K, Fukuoka M, Kawahara M, Nishikawa H, Takada Y, Kudoh S, et al. Phase III study of concurrent versus sequential thoracic radiotherapy in combination with mitomycin, vindesine, and cisplatin in unresectable stage III non-small-cell lung cancer. J Clin Oncol. 1999;17:2692-9.

4. Rusch VW, Albain KS, Crowley JJ, Rice TW, Lonchyna V, McKenna R Jr, et al. Surgical resection of stage IIIA and stage IIIB non-small-cell lung cancer after concurrent induction chemoradiotherapy. A Southwest Oncology Group trial. J Thorac Cardiovasc Surg. 1993;105:97-104. 
5. Rusch VW, Giroux DJ, Kraut MJ, Crowley J, Hazuka M, Johnson D, et al. Induction chemoradiation and surgical resection for non-small cell lung carcinomas of the superior sulcus: initial results of Southwest Oncology Group Trial 9416 (Intergroup Trial 0160). J Thorac Cardiovasc Surg. 2001;121:472-83.

6. Albain KS, Swann RS, Rusch VW, Turrisi AT 3rd, Shepherd FA, Smith C, et al. Radiotherapy plus chemotherapy with or without surgical resection for stage III non-small-cell lung cancer: a phase III randomised controlled trial. Lancet. 2009; 374:379-86.

7. Cerfolio RJ, Bryant AS, Jones VL, Cerfolio RM. Pulmonary resection after concurrent chemotherapy and high dose (60Gy) radiation for non-small cell lung cancer is safe and may provide increased survival. Eur J Cardiothorac Surg. 2009;35:718-23.

8. Sonett JR, Suntharalingam M, Edelman MJ, et al. Pulmonary resection after curative intent radiotherapy ( $>59$ Gy) and concurrent chemotherapy in nonsmall-cell lung cancer. Ann Thorac Surg. 2004;78:1200-5.

9. Bonner JA, Harari PM, Giralt J, Azarnia N, Shin DM, Cohen RB, et al. Radiotherapy plus cetuximab for squamous-cell carcinoma of the head and neck. $N$ Engl J Med. 2006;354:567-78.

10. Blumenschein GR Jr, Paulus R, Curran WJ, Robert F, Fossella F, WernerWasik M, et al. Phase II study of cetuximab in combination with chemoradiation in patients with stage IIIA/B non-small-cell lung cancer: RTOG 0324. J Clin Oncol. 2011;29:2312-8.

11. Govindan R, Bogart J, Stinchcombe T, Wang X, Hodgson L, Kratzke R, et al. Randomized phase II study of pemetrexed, carboplatin, and thoracic radiation with or without cetuximab in patients with locally advanced unresectable nonsmall-cell lung cancer: cancer and Leukemia Group B trial 30407. J Clin Oncol. 2011;29:3120-5.

12. Suntharalingam M, Paulus R, Edelman MJ, Krasna M, Burrows W, Gore E, et al. Radiation therapy oncology group protocol 02-29: a phase II trial of neoadjuvant therapy with concurrent chemotherapy and full-dose radiation therapy followed by surgical resection and consolidative therapy for locally advanced non-small cell carcinoma of the lung. Int J Radiat Oncol Biol Phys. 2012;84:456-63.

13. Edelman MJ, Hu C, Le Q, Donington JS, D'Souza WD, Dicker AP, et al. Randomized phase II study of preoperative chemoradiotherapy \pm panitumumab followed by consolidation chemotherapy in potentially operable locally advnced (stage IIIA, N2+) non-small cell lung cancer (LANSCLC): NRG oncology/ RTOG 0839. J Thorac Oncol. 2017;12:1413-20.

14. Roth JA, Fossella F, Komaki R, Ryan MB, Putnam JB Jr, Lee JS, et al. A randomized trial comparing perioperative chemotherapy and surgery with surgery alone in resectable stage IIIA non-small-cell lung cancer. J Natl Cancer Inst. 1994;86: 673-80.

15. Rosell R, Maestre J, Font A, Moreno I, Molina F, Milla A, et al. A randomized trial of mitomycin/ifosfamide/cisplatin preoperative chemotherapy plus surgery versus surgery alone in stage IIIA non-small cell lung cancer. Semin Oncol. 1994; 21:28-33.

16. Sher DJ, Fidler MJ, Seder CW, Liptay MJ, Koshy M. Relationship between radiation therapy dose and outcome in patients treated with neoadjuvant chemoradiation therapy and surgery for stage IIIa non-small cell lung cancer: a populationbased, comparative effectiveness analysis. Int J Radiat Oncol Biol Phys. 2015; 92:307-16

17. Guo SX, Jian Y, Chen YL, Cai Y, Zhang QY, Tou FF. Neoadjuvant chemoradiotherapy versus chemotherapy alone followed by surgery for resectable stage III non-small-cell lung cancer: a meta-analysis. Sci Rep. 2016;6: 34388.

18. Bharadwaj SC, Vallières E, Wilshire CL, Blitz M, Page B, Aye RW, et al. Higher versus standard preoperative radiation in the trimodality treatment of stage IIIA lung cancer. Ann Thorac Surg. 2015;100:207-13.

19. Martin J, Ginsberg RJ, Abolhoda A, Bains MS, Downey RJ, Korst RJ, et al. Morbidity and mortality after neoadjuvant therapy for lung cancer: the risks of right pneumonectomy. Ann Thorac Surg. 2001;72:1149-54.

20. Krasna MJ, Gamliel Z, Burrows WM, Sonett JR, Kwong KF, Edelman MJ, et al. Pneumonectomy for lung cancer after preoperative concurrent chemotherapy and high-dose radiation. Ann Thorac Surg. 2010;89:200-6.

21. Darling GE, Li F, Patsios D, Massey C, Wallis AG, Coate L, et al. Neoadjuvant chemoradiation and surgery improves survival outcomes compared with definitive chemoradiation in the treatment of stage IIIA N2 non-small-cell lung cancer. Eur J Cardiothorac Surg. 2015;48:684-90.

22. Eberhardt WE, Pöttgen C, Gauler TC, Friedel G, Veit S, Heinrich V, et al. Phase III study of surgery versus definitive concurrent chemoradiotherapy boost in patients with resectable stage IIIA(N2) and selected IIIB non-small-cell lung cancer after induction chemotherapy and concurrent chemoradiotherapy (ESPATUE). $J$ Clin Oncol. 2015;33:4194-201.

23. Pless M, Stupp R, Ris HB, Stahel RA, Weder W, Thierstein S, et al. Induction chemoradiation in stage IIIA/N2 non-small-cell lung cancer: a phase 3 randomised trial. Lancet. 2015;386:1049-56.

24. LaPar DJ, Bhamidipati CM, Lau CL, Jones DR, Kozower BD. The Society of Thoracic Surgeons General Thoracic Surgery Database: establishing generalizability to national lung cancer resection outcomes. Ann Thorac Surg. 2012;94:216-21.

25. Fernandez FG, Kosinski AS, Burfeind W, Park B, DeCamp MM, Seder C, et al The Society of Thoracic Surgeons Lung Cancer Resection Risk Model: higher quality data and superior outcomes. Ann Thorac Surg. 2016;102:370-7.

26. Cho JH, Kim J, Kim K, Shim YM, Kim HK, Choi YS. Risk associated with bilobectomy after neoadjuvant concurrent chemoradiotherapy for stage IIIA-N2 non-small-cell lung cancer. World J Surg. 2012;36:1199-205.

27. Farjah F, Backhus L, Cheng A, Englum B, Kim S, Saha-Chaudhuri P, et al. Failure to rescue and pulmonary resection for lung cancer. J Thorac Cardiovasc Surg. 2015;149:1365-71

28. Brunelli A, Xiume F, Al Refai M, Salati M, Marasco R, Sabbatini A. Riskadjusted morbidity, mortality and failure-to-rescue models for internal provider profiling after major lung resection. Interact Cardiovasc Thorac Surg. 2006;5: 92-6.

29. Lv W, Zhang GQ, Jiao A, Zhao BC, Shi Y, Chen BM, et al. Chemotherapy plus cetuximab versus chemotherapy alone for patients with KRAS wild type unresectable liver-confined metastases colorectal cancer: an updated meta-analysis of RCTs. Gastroenterol Res Pract. 2017;2017:8464905.

30. Niyazi M, Maihoefer C, Krause M, Rodel C, Budach W, Belka C. Radiotherapy and "new" drugs-new side effects? Radiat Oncol. 2011;6:177.

31. Upérin A, Le Péchoux C, Rolland E, Curran WJ, Furuse K, Fournel P, et al. Metaanalysis of concomitant versus sequential radiochemotherapy in locally advanced non-small-cell lung cancer. J Clin Oncol. 2010;28:2181-90.

32. Gore E, Hu C, Bar-Ad V, Robinson CG, Wheatley MD, Bogart JA, et al. Impact of incidental cardiac irradiation on cardiopulmonary toxicity and survival for locally advanced NSCLC: reanalysis of NRG Oncology RTOG 0617 with centrally contoured cardiac structures. Int J Radiat Oncol. 2017;96(Suppl):S129-30.

33. Wang K, Eblan MJ, Deal AM, Lipner M, Zagar TM, Wang Y, et al. Cardiac toxicity after radiotherapy for stage III non-small-cell lung cancer: pooled analysis of dose-escalation trials delivering 70 to 90 Gy. J Clin Oncol. 2017;35: 1387-94.

34. Steuer CE, Behera M, Ernani V, Higgins KA, Saba NF, Shin DM, et al. Comparison of concurrent use of thoracic radiation with either carboplatin-paclitaxel or cisplatin-etoposide for patients with stage III non-small-cell lung cancer: a systematic review. JAMA Oncol. 2017;3:1120-9.

35. Santana-Davila R, Devisetty K, Szabo A, Sparapani R, Arce-Lara C, Gore EM et al. Cisplatin and etoposide versus carboplatin and paclitaxel with concurren radiotherapy for stage III non-small-cell lung cancer: an analysis of Veterans Health Administration data. J Clin Oncol. 2015;33:567-74.

Key Words: lung cancer, induction therapy, surgery, radiation, chemotherapy

\section{Discussion}

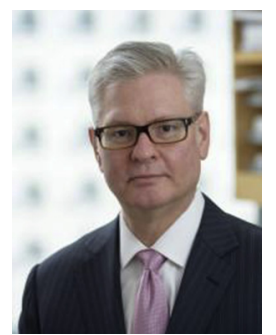

Dr David R. Jones (New York, NY). I have no disclosures. I would like to congratulate Dr Donington and her co-authors for an excellent paper and a superb presentation that examines essentially the safety of surgical resection following induction therapy with higher dose radiation in this highly selected group of patients with pathologically proven $\mathrm{N} 2$ non-small cell lung cancer. By combining 2 small prospective studies, the authors attempt to address a perceived fear 
of increased morbidity and/or mortality associated with the use of radiation in the induction setting. I have 3 questions for Dr Donington and I will ask them individually.

Despite combining these trials, there are still only 93 patients in the analysis and 77 of these had a lobectomy. There was a significant increase in 30- and 90-day mortality in the 16 patients who had more than a straightforward lobectomy. Specifically, mortality was $19 \%$ at 90 days in those 5 patients who had a pneumonectomy, 5 patients with a bilobectomy and in the 2 patients with sleeve resections. How do you explain this increased mortality, what was the cause of death, and do you believe it is related to the induction regimen?

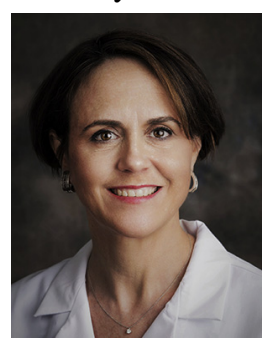

Dr Jessica S. Donington. We were a little surprised by the pattern of death; it was quite variable. There was a bronchopleural fistula after a pneumonectomy, but also someone who died from complications of a necrotic muscle flap, and one with pulmonary edema, so we saw this wide variety. We believe that the deaths may have been more a result of failure to rescue. Maybe it wasn't really directly our therapy but the fact that these patients had had induction therapy, be that radiation or chemo, and then went on to this bigger operation, overall it was difficult for them to overcome any complication.

Dr Jones. My second question relates to work from our group and others who have shown that a predicted postoperative diffusion capacity is associated with increased perioperative morbidity and mortality. In your manuscript and in your slides you apparently only looked at forced expiratory volume in 1 second. However, there are many studies that have show diffusion capacity is a predictor of increased morbidity in this patient population. Was that data element available to you in your analysis?

Dr Donington. No, it wasn't, unfortunately. In the 0229 trial, which was designed many years ago, I don't know that we all appreciated the value of diffusion capacity for carbon monoxide at that point. But I do agree going forward that would be important because it would also tie in the extent of resection and how much lung you were taking, and I agree going forward we discussed that as a preoperative factor that would need to be included.

Dr Jones. My final question really relates to the need to include radiation at all as part of the induction regimen. It is often difficult, if not impossible, for the surgeon to know the radiation volumes and doses and, more importantly, whether an extended resection may be needed, which is often is an intraoperative decision. Given the results of the prospective Swiss study where no additional benefit was observed with radiation relative to chemotherapy alone for overall or disease-free survival, would it not be reasonable to consider treating patients with induction chemotherapy alone and then using radiation in an adjuvant setting for persistent $\mathrm{N} 2$ disease or perhaps for an R1 or an $\mathrm{R} 2$ resection? I realize that in the Swiss Group for Clinical Cancer Research study the radiation was delivered sequentially and at a slightly lower dose of radiation, like 41 Gy. Can your study help me understand why this wouldn't be a preferred approach, particularly given the high mortality of $19 \%$ in this subset of patients?

Dr Donington. Thank you. I think there are a couple of important concepts. The first, which is important to understand, is that the treatment we gave our patientschemotherapy and concurrent full dose radiation-is what patients get who are nonoperative. We gave them the best care out there, and for many institutions that is the standard of care. What we said is that surgery can improve survival above that if we can perform it safely. So I think that that difference is important. Giving patients sequential therapy does not provide the same level of mediastinal nodal clearance or pathologic complete response, and we know that both in the operative and nonoperative populations.

So I think this is an important approach to treatment, not only because it is where my group believes the best survival will be in those we try to treat prospectively but also for those young patients who are initially treated without a plan for surgery somewhere else and then present to our clinics. But I also think it is a dangerous combination if you think you are going to do an extended resection.

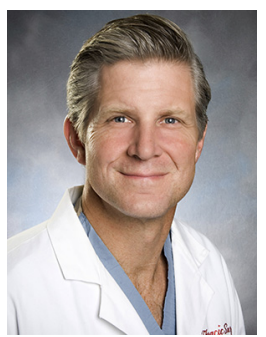

Dr Scott Swanson (Boston, Mass). Jessica, that was an excellent talk, and I agree with you that surgery is necessary to improve survival in stage IIIA, because chemo and radiation alone really doesn't do it. So my question is, with the conclusions you found, what are your recommendations about using induction targeted therapy and what are you going to do going forward with the extended resection, how do you use this data in your practice?

Dr Donington. In our practice currently for a patient who we believe-and I believe you can tell pretty well up frontand who we think is going to get a lobectomy, we treat them like this: a trimodality approach with chemotherapy, radiation, and resection. If I have a patient who looks like they are going to require a pneumonectomy or a bilobectomy, depending we will either treat them with induction chemotherapy alone followed by resection and the option for radiation after or definitive chemotherapy and radiation alone if we don't feel that they are a strong surgical candidate.

I believe this whole playing field is about to change as we now are starting to look at checkpoint inhibitors in the stage III setting. 


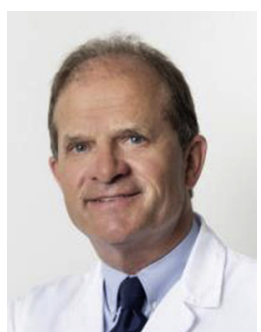

tomies was below $1 \%$.

Dr Donington. Dr Weder, I knew I would be talking with you this morning. That is a wonderful trial that you participated in and the Europeans have done a beautiful job with these very complex treatment regimens. I think that an operative mortality after these complex resections of $4 \%$ isn't extraordinary, but $1.3 \%$ for lobectomy is, and I am going to stand by that data. The difference between 1.3 and 1 or 2 is very small and I think these are really good results, as good as what you published from Europe.

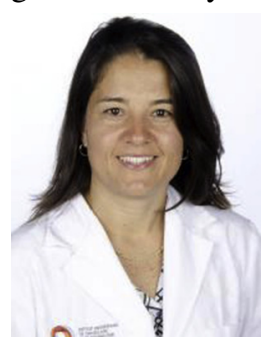

Dr Paul A. Ugalde (Québec City, Quebec, Canada). Jessica, congratulations and great work. Yesterday we spent a lot of time discussing the management of $\mathrm{N} 2$ patients. I just wanted to understand again your concept on this. What you are telling us is that actually the ideal treatment for these patients is induction chemoradiation therapy as suggested by oncologists and radiotherapists and that we can add surgery to some of those patients. So as a concept is that what you are proposing?

Dr Donington. Yes, I believe we can add surgery to a majority of those patients. Again, anyone who I think doesn't require a bilobectomy or a pneumonectomy could safely have surgery added, and I believe that is their best chance for care. We might know for sure in 5 years or so when our data matures.

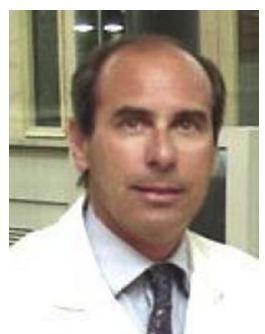

Dr Laureano Molins (Barcelona, Spain). I enjoyed very much your presentation. What is not clear to me is did you restage the patients and some of them you just refused because they were pathological N2 but the others went into operation? So it is not clear to me what is the rule to discharge prior or not the patients for surgery.

Dr Donington. As the investigators, we couldn't agree either. The pre-resection invasive staging was done at the discretion of each institution. So not all patients underwent preoperative pathologic reassessment of their mediastinum. But for those institutions where that was their choice, then they did not bring those patients with residual nodal disease on to resection. That approach was the minority in this trial, where most patients were taken directly to resection. And I do hope to look at outcomes between those two groups at a later time, because now we have persistent $\mathrm{N} 2$ patients who were treated without surgery and those that went on, and that is an area of controversy still in our community.

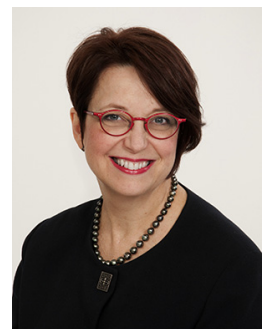

Dr Gail E. Darling (Toronto, Ontario, Canada). Jessica, that was fabulous. Thank you so much for bringing this forward and putting the data together. I had a question about the persistent $\mathrm{N} 2$ as well, because in the intergroup 0139, although the patients who had mediastinal sterilization had the best survival, there was improved survival even in those who had persistent $\mathrm{N} 2$, and going forward what would your recommendation be?

Dr Donington. As I said, I am working now to be able to do that analysis, because I still think that surgery is really important for that group of patients, and I don't like staging them and not bringing them to resection. I think that is the next analysis we need to do. This is unfortunately a fairly small subset that we are going to be looking at, 7 versus 20 or so, but hopefully it gives us at least some insight of where to go forward, because I don't think we have a lot of data in this area.

Dr Darling. What would your recommendation be to those of us in the room?

Dr Donington. I think that these patients should go to resection. A survival of $10 \%$ or $15 \%$, which is what the literature tells us, is better than a survival of 0 , which is what happens to those who don't go on to resection.

Dr Jones. I again congratulate you, as the other discussants have, on bringing this to our attention. You have clearly been able to demonstrate that thoracic surgeons can do a lobectomy after chemotherapy and radiation and it can be done safely. The real challenge is what do we do with that high mortality of $19 \%$ at 90 days in patients who get more than that and how do we better select those people up front? I also agree that the best treatment for persistent $\mathrm{N} 2$ disease is to remove the persistent N2 disease, and that means they need an operation.

Dr Donington. Thank you very much for your comment. I think we can choose these patients. And the $19 \%$ mortality, again, you are forgetting about that epidermal growth factor receptor antibody. It was really troublesome and none of us appreciated the toxicity that this causes in the lung, but we also saw this in an Radiation Therapy Oncology Group trial that didn't involve surgery (RTOG 0617). So although it is used in the head and neck and colon, it is probably not a good agent in lung and taking that out may have reduced our toxicity. 
TABLE E1. Radiation plan data for 0839 patients $(n=54)$

\begin{tabular}{|c|c|c|c|c|c|}
\hline Plan & Ipsilateral lung GTV & Contralateral lung & Combined lung GTV & Heart & Esophagus \\
\hline $\mathrm{V}_{5}$ & 68.2 & 44.3 & 56.5 & & \\
\hline $\mathrm{V}_{10}$ & 61.2 & 20.6 & 41.1 & 25.3 & \\
\hline $\mathrm{V}_{20}$ & 49.4 & 3.8 & 28.8 & 16.5 & \\
\hline $\mathrm{V}_{30}$ & 38.1 & 0.7 & 20.9 & 11.3 & \\
\hline $\mathrm{V}_{45}$ & & & & 5.1 & \\
\hline $\mathrm{V}_{60}$ & & & & 0.7 & \\
\hline Mean dose (Gy) & 24.55 & 6.15 & 16.10 & & 23.25 \\
\hline Max dose (Gy) & & & & & 63.14 \\
\hline
\end{tabular}

